GAPP, número 24, noviembre de 2020

Sección: ESTUDIOS

Recibido: 15-06-2020

Modificado: $16-10-2000$

Aceptado: $19-10-2000$

DOI: https://doi.org/10.24965/gapp.i24.10811

Páginas: 6-23

\title{
La Administración tras el coronabreak. Políticas para ¿un nuevo paradigma administrativo?
}

\section{The Administration after the coronabreak. Policies for a new administrative paradigm?}

\author{
Óscar Cortés Abad \\ Ayuntamiento de Madrid (España) \\ ORCID: https://orcid.org/0000-0002-6657-5582 \\ oscarcortes99@gmail.com
}

\section{NOTA BIOGRÁFICA}

Doctor en Derecho por la Universidad de A Coruña e Ingeniero Industrial del ICAI. Profesor en distintas universidades y escuelas de negocios e investigador del Observatorio para la Transformación del Sector Público ESADE-PwC. Ha sido asesor del ministro de Justicia entre 2014 y 2018 y desempeñado otros cargos en el sector público estatal. Actualmente dirige la comunicación digital del Ayuntamiento de Madrid.

\section{RESUMEN}

El presente trabajo ${ }^{1}$ tiene por objeto estudiar el impacto de la COVID-19 en las políticas de reforma administrativa estableciéndose la hipótesis de que esta será una crisis disruptiva que dará impulso a un nuevo ciclo de reformas. Se trata de un estudio descriptivo y exploratorio que tiene como marco conceptual los efectos que los cambios en el entorno producen sobre las políticas destinadas a reformar el subsistema institucional. Para ello comenzaremos repasando algunos hitos históricos y su relación con la evolución del paradigma administrativo. A continuación, se analizará el contexto previo al coronabreak y las políticas que venían desarrollándose hasta ese momento, para posteriormente estudiar las decisiones más relevantes tomadas durante los primeros seis meses de pandemia en la Administración General del Estado y sus efectos más inmediatos sobre el subsistema institucional. Finalmente, la discusión se centra en cómo estas medidas van a impactar en las políticas que se venían desarrollando y las debilidades encontradas, concluyendo que las acciones de reforma más inmediatas se articularán sobre dos conjuntos de políticas: políticas de transformación y políticas de fortalecimiento institucional.

\section{PALABRAS CLAVE}

COVID-19; reforma administrativa; políticas de transformación; políticas de fortalecimiento; entornos disruptivos.

\section{ABSTRACT}

The work aims to study the impact of COVID-19 on administrative reform policies, establishing the hypothesis that this will be a disruptive crisis that will give impetus to a new cycle of reforms. This is a descriptive and

1 El autor agradece a los editores de la revista por la publicación de este estudio, así como el trabajo realizado por los evaluadores anónimos que lo han revisado proponiendo aportaciones de un inestimable valor que han servido para enriquecerlo. 
exploratory study whose conceptual framework is the effects that changes in the environment produce on the policies aimed at reforming the institutional subsystem. For this, we will begin by reviewing some historical milestones and their relationship with the evolution of the administrative paradigm. Next, the context prior to the coronabreak and the policies that had been developed up to that moment will be analyzed, to later study the most relevant decisions taken during the first six months of the pandemic and their most immediate effects on the institutional subsystem. Finally, the discussion focuses on how these measures are going to impact the policies that were being developed and the weaknesses found, concluding that the most immediate reform actions will be based on two sets of policies: transformation policies and institutional strengthening policies.

\section{KEYWORDS}

COVID-19; administrative reform; transformation policies; strengthening policies; disruptive context.

\section{SUMARIO}

INTRODUCCIÓN. 1. EL IMPACTO DEL ENTORNO EN LAS POLITICAS DE REFORMAADMINISTRATIVA: ALGUNAS COORDENADAS HISTÓRICAS. 2. EL CONTEXTO DE DISRUPCIÓN LATENTE Y LAS POLÍTICAS DE REFORMA ANTES DEL CORONABREAK. 2.1. FACTORES DE ENTORNO. 2.2. POLÍTICAS DE REFORMA. 3. LA COVID-19 COMO ACELERADOR DE LA DISRUPCIÓN DEL ENTORNO. 4. FASES DE LA CRISIS DE LA COVID-19 Y DECISIONES PÚBLICAS MÁS RELEVANTES. 5. LA ADMINISTRACIÓN TRAS EL CORONABREAK. DISCUSIÓN Y PROPUESTAS. CONCLUSIONES. REFERENCIAS BIBLIOGRÁFICAS.

\section{INTRODUCCIÓN}

Las políticas públicas son el conjunto de decisiones gubernamentales que pretenden dar respuesta a las necesidades de una sociedad (Navarro, 2008) incluyéndose, dentro de ese conjunto, sus contenidos, los mecanismos para hacerlas efectivas y la evaluación de los impactos que producen.

Según Salazar (2009) toda política pública consta de tres subsistemas: el político, donde se toman las decisiones; el social, donde surgen las necesidades a atender; y el administrativo, que ejecuta la política. Afirma Villoria (2011: 31) que «no puede haber buen gobierno, ni buena sociedad, sin las estructuras institucionales adecuadas» por lo que la Administración, como núcleo de dicho subsistema administrativo, es fundamental para el éxito de acción de gobierno y objeto de políticas de reforma que persigan maximizar su eficacia.

La literatura científica se refiere de manera frecuente al impacto del entorno en las políticas públicas, entendiendo por entorno lo que Mintzberg (1984) definió como todo aquello que es ajeno la organización. Lasswell y Lerner (1951) fueron de los primeros en reconocer que el contexto es parte fundamental del proceso de diseño de la política pública. Kuhn (1970) hizo hincapié en el componente socio histórico, del que el contexto es parte fundamental, para enfocar adecuadamente la acción gubernamental. Berman (1980) y Mayntz (1980) se refieren a las denominadas variables de situación que determinan un contexto (organizativo, político, social y legal), las cuales inciden en las metas de la política, la cantidad y posición relativa de los actores respecto a la misma, el grado de resistencia de los encargados de su implementación o el nivel de control ejercido por los estamentos superiores. Las variables de situación tienen relación con las condiciones socioeconómicas o tecnológicas, pero también con otros factores como la atención que los medios de comunicación prestan al problema o el clima en la opinión pública (Sabatier y Mazmanian, 1981).

Adaptando a Mintzberg (1984) a la hora de analizar los entornos podríamos señalar cuatro factores que los caracterizan. En primer lugar la volatilidad, que tiene que ver con la fugacidad de los acontecimientos, la velocidad con la que se producen, la poca fijeza de las cosas; en segundo lugar, la complejidad, que responde al carácter más simple o compuesto de aspectos que lo determinan y los actores implicados; en tercer lugar, la incertidumbre, que retrata cómo va a evolucionar, si de manera más previsible o de manera más incierta; por último, la ambigüedad, que mediría la claridad para poder discernir o anticipar cuáles van a ser sus características más importantes.

A partir de estos cuatro factores se pueden establecer diversas tipologías de entornos. Continuando con Mintzberg (1984) los entornos estables se caracterizarían por una baja volatilidad, simplicidad de los 
atributos que los componen, alta previsibilidad en la evolución de los acontecimientos y claridad a la hora de definirlos. En un punto intermedio se situarían los que podríamos llamar entornos reactivo-adaptativos, que cambian y evolucionan, pero lo hacen lentamente, tienen algo más de complejidad e incertidumbre y presentan una cierta, aunque baja, ambigüedad. Por último, los entornos disruptivos, también llamados VUCA ${ }^{2}$ son aquellos inestables, inciertos, complejos y ambiguos. A su vez, estos entornos disruptivos pueden ser categorizados según los cambios estén más o menos acotados en el tiempo (corta o larga duración) o si dichos cambios se producen de manera abrupta (radical) o gradual.

Nuestro estudio tiene por hipótesis considerar que la crisis de la COVID-19 es un cambio disruptivo de tal envergadura que puede impulsar un nuevo ciclo de políticas de reforma administrativa. Para contrastar dicha hipótesis hemos seguido un método cualitativo que recurre a la interpretación de información obtenida de estos primeros seis meses de pandemia, en los que, por la novedad del fenómeno y a falta de estudios sistemáticos de la materia, aún es prematuro disponer de datos precisos y en suficiente cantidad como para plantear otro tipo de modelos. Este enfoque cualitativo se considera sistemático y científico para procesos complejos donde son valorables el intangible asociado a la experiencia o al análisis de pautas de actuación (Keohane, King y Verba, 2000).

Se trata por tanto de un estudio descriptivo y exploratorio sobre el impacto de la COVID-19 en la Administración y en las políticas para su reforma concluyéndose que tras la pandemia habrá políticas de las que se venían desarrollando que se van a acelerar y los primeros pasos de dicha aceleración se habrían dado en estos meses (la digitalización), otras que se van a modificar (el ajuste por un fortalecimiento de los servicios públicos) y otras nuevas que pasarán a ser desarrolladas en próximos años (una reforma del empleo público que se antoja ineludible).

Para ello, en un primer capítulo se analizan los antecedentes que muestran cómo hitos históricos han dado lugar a factores de entorno condicionantes de las políticas de reforma administrativa. A continuación, pasamos a analizar el entorno pre COVID-19 y las políticas puestas en marcha en ese periodo. Posteriormente se desgranan las medidas más importantes puestas en marcha durante los primeros seis meses de pandemia y su impacto en la Administración pública recurriendo para ello a fuentes de información de la actualidad (blogs, noticias, etc.) así como a otros estudios realizados en este periodo. Por último, se analizan estas medidas y cómo pueden afectar a las políticas de reforma administrativa que se venían desarrollando o a las nuevas que se puedan poner en marcha.

\section{EL IMPACTO DEL ENTORNO EN LAS POLÍTICAS DE REFORMA ADMINISTRATIVA. ALGUNAS COORDENADAS HISTÓRICAS}

Las políticas de reforma administrativa no son una excepción y uno de los factores que las condicionan son los cambios en el entorno que se han venido produciendo. Mendoza y Vernis (1995: 144) nos señalan que «el papel del Estado contemporáneo ha ido evolucionando a medida que ha cambiado la sociedad y el mercado» de tal manera que en dicho rol tienen incidencia las circunstancias de entorno económico-social y político que se produzcan, correspondiendo a cada modelo de Estado, a su vez, un determinado modelo de Administración pública. Echebarría (1998: 93) incide en que es necesario «responder a los retos de un entorno cada vez más amplio y complejo» para justificar el desarrollo evolutivo que se ha ido produciendo a lo largo de las décadas en el modelo administrativo.

La Revolución Francesa y la Revolución Industrial son los dos sucesos más relevantes que condicionan el devenir histórico a partir de finales del siglo XVIII y durante todo el siglo XIX. Como resultado de ambos acontecimientos se produce el proceso de construcción jurídico-político de los Estados Nación que, a su vez, da lugar al nacimiento del sujeto que es objeto de las políticas a las que se refiere este estudio, la Administración pública como subsistema que adopta la burocracia, considerada por Olmeda (1999: 276), como «el modelo científico de la organización del trabajo más influyente en el pensamiento organizativo contemporáneo».

Un segundo hito relevante es el contrato social nacido tras la Segunda Guerra Mundial que da lugar a las políticas bienestar. Un hecho que da pie a un nuevo ciclo de reforma administrativa que lleva a las Administraciones a convertirse en organizaciones de servicios, abriéndose la posibilidad de que no sólo

\footnotetext{
2 VUCA, acrónimo en inglés, de volátil, incierto, complejo y ambiguo.
} 
GAPP. Nueva Época - N. ${ }^{2}$ 24, noviembre 2020 - ISSN: 1989-8991 - DOI: https://doi.org/10.24965/gapp.i24.10811 - [Págs. 6-23]

actúen como burocracias aplicadoras del principio de legalidad, sino también como prestadoras de servicios públicos.

El tercer hito que no queremos pasar por alto son las sucesivas crisis del petróleo de finales del siglo pasado, que dan lugar a una crisis social y económica que marca el inicio de un nuevo ciclo de políticas de reforma destinadas a llevar la Administración a un nuevo escenario post burocrático (Barzelay, 1998). La nueva gestión pública nace como paradigma de reinvención del gobierno (Gaebler y Osborne, 1992) para orientar a la Administración pública hacia principios de eficacia, eficiencia o economía de recursos.

En cuarto lugar nos referimos al fenómeno de la globalización, gracias al cual se produce el despertar de un espacio público no estatal (Valenzuela, 2013) que pone de manifiesto la necesidad de replantear el rol del Estado como parte de un ecosistema más amplio que considere el gran número de actores presentes en la sociedad. La respuesta es la gobernanza relacional como paradigma que alude a «un conjunto de mecanismos, procesos, instituciones e incluso valores compartidos mediante los cuales los ciudadanos y grupos sociales articulan sus intereses y diferencias para el ejercicio de sus derechos y obligaciones» (Grasa, 2005: 88).

En todos los casos anteriores encontramos un elemento en común: se trata de hechos históricos que dan lugar a entornos disruptivos en los que se produce una ruptura con el statu quo que, a su vez, desencadena un replanteamiento de la acción pública y del instrumento principal que sirve para ejecutarla, el subsistema administrativo.

TABla 1. Cambios en el entorno y Ciclo de Reforma ADministrativa

\begin{tabular}{lll}
\hline \multicolumn{1}{c}{ Hito histórico } & \multicolumn{1}{c}{ Tipo de cambio } & \multicolumn{1}{c}{ Paradigma de reforma } \\
\hline Revolución Francesa. & Disruptivo de corta duración y radical. & Nacimiento de la Administración burocrática. \\
\cline { 1 - 2 } Revolución Industrial. & Disruptivo de larga duración y gradual. & \\
\cline { 1 - 2 } $\begin{array}{l}\text { Segunda Guerra } \\
\text { Mundial. }\end{array}$ & Disruptivo de corta duración y radical. & $\begin{array}{l}\text { Administración como organización de } \\
\text { servicios. }\end{array}$ \\
\hline $\begin{array}{lll}\text { Sucesivas crisis } \\
\text { del petróleo. }\end{array}$ & Disruptivo de larga duración y gradual. & $\begin{array}{l}\text { Administración post burocrática } \\
\text { Nueva gestión pública. }\end{array}$ \\
\hline Globalización. & Disruptivo de larga duración y gradual. & Gobernanza relacional. \\
\hline
\end{tabular}

Fuente: Elaboración propia.

\section{EL CONTEXTO DE DISRUPCIÓN LATENTE Y LAS POLÍTICAS DE REFORMA ANTES DEL CORONABREAK}

\subsection{Factores de entorno}

Llegado el siglo XXI se suceden una serie de factores de entorno que determinan las políticas de reforma administrativa puestas en marcha en estas dos primeras décadas.

a) La revolución tecnológica, revolución digital o cuarta revolución industrial (Schwab, 2016): nace con la universalización de Internet, su aplicación práctica a modo de red de empresas (Dans, 2010). A partir de este momento se superponen tecnologías como las llamadas tecnologías sociales que permiten conectar a usuarios para coproducir contenidos, colaborar y participar. La conectividad lleva a que el dato se convierta en un nuevo activo, la "sangre de la economía» (O'Reilly, 2011: 14) ${ }^{3}$ y en un auténtico quebradero de cabeza por las múltiples controversias y perjuicios que su utilización indebida puede ocasionar a sus legítimos titulares.

Otras tecnologías más recientes, y por tanto menos maduras, como el internet de las cosas, el almacenamiento en la nube, el blockchain o la inteligencia artificial condicionan de manera significa-

3 También llamado petróleo del siglo XXI, cita célebre que se atribuye originalmente al matemático británico Clive Humby. 
tiva la prestación de servicios, el funcionamiento interno de las organizaciones y las relaciones entre éstas y sus clientes o consumidores.

b) La consolidación fiscal: la llamada Gran Recesión que tuvo lugar en España entre 2008 y 2013 afloró elevados desequilibrios de las cuentas públicas en determinados Estados que, como España, vieron reducidos drásticamente sus ingresos públicos ${ }^{4}$. Los altos niveles de déficit público acumulados durante los años de crisis derivaron en políticas de consolidación fiscal cuyo objetivo principal fue contener el fuerte endeudamiento, agravado a su vez por el retroceso del Producto Interior Bruto ${ }^{5}$ debido a la caída del consumo y la inversión.

c) La desconfianza en lo público: otro aspecto relevante en este contexto es la brecha de confianza entre ciudadanía e instituciones que vienen detectando diferentes estudios de opinión ${ }^{6}$. Villoria (2016) apunta a los efectos de la Gran Recesión como causa de que el grado de desconfianza en los poderes públicos sea muy superior en España respecto a sus homólogos europeos. Otro de los motivos, señalado por Acemoglu y Robinson (2012), podría ser la caída de los modelos de bienestar que afectan a unos países desarrollados, especialmente del continente europeo, en los que la dimensión social ha sido parte fundamental del contrato vertebrador de la sociedad. También las prácticas de las denominadas élites extractivas (Molinas, 2013), condensadas en la catarata de casos de corrupción política que en muy poco tiempo han llevado a que este asunto escale posiciones en las preocupaciones de los españoles.

d) La multiplicación de los riesgos: el World Economic Forum, en uno de sus últimos informes Global Risks Report 2019, describe diferentes riesgos que condicionan el entorno o previo a la crisis desatada por la pandemia. Riesgos económicos como los desequilibrios macro ocasionados por las consecuencias de la globalización financiera, las pulsiones proteccionistas, los elevados déficits fiscales, etc. Riesgos medioambientales como la huella del hombre sobre el territorio, la tensión sobre recursos naturales, alteraciones sobre el clima por el calentamiento global. Riesgos geopolíticos como el terrorismo internacional, la existencia de Estados fallidos o el desplazamiento del centro de gravedad del poder político y económico a la región Asia Pacífico. Riesgos sociales como crisis alimentarias, las migraciones o extensión de enfermedades infecciosas. Riesgos tecnológicos como aquellos que desde el propio ciberespacio amenazan a sistemas críticos o infraestructuras de comunicaciones.

e) La fragmentación social, política e institucional: el entorno previo al coronabreak se caracteriza también por una fragmentación de los grupos que dirigen, actúan e influyen sobre los asuntos públicos. Si hasta hace bien poco los actores eran pocos, previsibles, fácilmente identificables y con fuerza representativa, hoy el universo tiende a atomizarse. El modelo de Estado compuesto, con una marcada descentralización competencial, las tensiones identitarias y la pluralidad de fuerzas políticas que han adquirido representación institucional hace complejo e inestable el contexto para las políticas públicas.

\subsection{Políticas de reforma administrativa}

Todos estos factores de entorno son causa determinante de las políticas públicas de reforma administrativa más relevantes que se han ido poniendo en marcha en los últimos años.

\section{Las políticas de digitalización del sector público}

Llegan como consecuencia de la necesaria adaptación a un contexto más digital sujeto a restricciones presupuestarias. Este planteamiento de modernizar el Estado weberiano usando la tecnología impulsa el cambio en una doble dirección. La externa, o lo que Margetts y Dunleavy (2013) han venido a denominar

4 Entre 2007 y 2013, España fue el país que sufrió la mayor caída de ingresos públicos por habitante, un $3 \%$, frente a otros como Grecia $(2 \%)$ e Irlanda $(2,5 \%)$.

5 Entre 2008 y 2013 , el PIB español se contrajo un 5,8\%.

6 La Organización para la Cooperación y el Desarrollo Económico (OCDE, 2017) ha situado a España a la altura de México, Hungría o Portugal en cuanto a tasa de percepción de corrupción (alta) y confianza en el gobierno (baja). El último barómetro del Centro de Investigaciones Sociológicas (CIS, 2020) señala que «el mal comportamiento de los políticos» es el cuarto problema que más preocupa a los españoles (para un $23,1 \%$ ) mientras que «los problemas políticos en general» es el sexto (para un 17,0\%). 
digital era government, que tiene que ver con el impacto de lo digital en la intermediación de la Administración pública con la ciudadanía, lo cual a su vez abre espacios de autoorganización y creación de valor al margen del aparato administrativo (gobierno como plataforma de O'Reilly (2011) o do-it-yourself-government de Margertts y Dunleavy (2013)).

Y la perspectiva interna que consiste en digitalizar el funcionamiento burocrático bajo una concepción de servicios, eficiencia y agilidad propios de la nueva gestión pública (Brown, 2005) y en la línea marcada por el World Economic Forum (2011) de avanzar hacia un Estado FAST, acrónimos en inglés de flat, agile, streamlined and techdriven.

TABLA 2. LAS TECNOLOGÍAS DE LA INFORMACIÓN COMO ACOMPAÑANTES DEL PROCESO DE REFORMA DEL SECTOR PÚBLICO

\begin{tabular}{|c|c|c|c|}
\hline $\begin{array}{c}\text { Modelo } \\
\text { tecnológico }\end{array}$ & $\begin{array}{l}\text { Papel de la } \\
\text { tecnología }\end{array}$ & Algunos hitos de aplicación en España & Algunos resultados \\
\hline e-government & $\begin{array}{l}\text { Digitalizar } \\
\text { procedimientos } \\
\text { existentes. } \\
\text { Nuevos servicios } \\
\text { digitales. } \\
\text { Eficiencia, } \\
\text { descentralización } \\
\text { e interoperabilidad. }\end{array}$ & $\begin{array}{l}\text { El nuevo procedimiento digital a través de } \\
\text { la Ley } 39 / 2015 \text {, de } 1 \text { de octubre, del Proce- } \\
\text { dimiento Administrativo de las Administracio- } \\
\text { nes Públicas. } \\
\text { Plan de Transformación Digital de la Adminis- } \\
\text { tración General del Estado. } \\
\text { La digitalización obligada del procedimiento } \\
\text { judicial a través de la ley } 42 / 2015 \text {, de } 5 \text { de } \\
\text { octubre, de reforma de la ley } 1 / 2000 \text {, de } 7 \text { de } \\
\text { enero, de Enjuiciamiento Civil. }\end{array}$ & $\begin{array}{l}\text { Avance desigual y heterogé- } \\
\text { neo. Ninguna de las Admi- } \\
\text { nistraciones cumple con la } \\
\text { totalidad de requerimientos } \\
\text { extraídos de las leyes } 39 \text { y } \\
40 / 2015 \text {. }\end{array}$ \\
\hline gov 2.0 & $\begin{array}{l}\text { Facilitar } \\
\text { la relación } \\
\text { con los } \\
\text { ciudadanos. }\end{array}$ & $\begin{array}{l}\text { Resolución de } 21 \text { de marzo de } 2013 \text {, de la } \\
\text { Secretaría de Estado de Administraciones } \\
\text { públicas, por la que se aprueba la Guía de } \\
\text { Comunicación Digital para la Administración } \\
\text { del Estado. } \\
\text { Guías y normas de uso de las redes sociales } \\
\text { en las Comunidades Autónomas y Adminis- } \\
\text { tración Local. }\end{array}$ & $\begin{array}{l}\text { Presencia generalizada en } \\
\text { redes sociales de la Adminis- } \\
\text { tración General del Estado, } \\
\text { Administración Autonómica y } \\
\text { Administración Local (Cortés, } \\
\text { 2019; Criado y Rojas, 2015a; } \\
\text { Criado y Rojas, 2015b). }\end{array}$ \\
\hline $\begin{array}{l}\text { gov as a data, } \\
\text { smart gov }\end{array}$ & $\begin{array}{l}\text { Toma de } \\
\text { decisiones, } \\
\text { coproducción, } \\
\text { empoderamiento, } \\
\text { innovación. }\end{array}$ & $\begin{array}{l}\text { Estrategia Española de I+D+I en Inteligencia } \\
\text { Artificial. } \\
\text { Plan de Impulso de las Tecnologías del Len- } \\
\text { guaje. } \\
\text { Creación de Secretaría de Estado de Digitali- } \\
\text { zación e Inteligencia Artificial. }\end{array}$ & Por desarrollar. \\
\hline
\end{tabular}

Fuente: Elaboración propia.

\section{Las políticas de ajuste administrativo}

Resultan de la aplicación al sector público de las pautas de consolidación fiscal derivadas de la crisis presupuestaria que tuvo lugar durante la Gran Recesión. Estas políticas de ajuste, también llamadas de austeridad, se manifestaron en la fijación de límites al gasto público y refuerzo del control con impacto directo en servicios esenciales, empleo y organización administrativa.

Algunas de las políticas de ajuste administrativo puestas en marcha en España durante la segunda década del siglo XXI son las siguientes:

a) Las políticas de ajuste en los servicios esenciales: concretamente en sanidad, por su relación directa de la crisis de la COVID-19, y según Bandrés y González (2015) el esfuerzo de reducción del gas- 
to sanitario alcanzó un $12 \%$ concentrándose en caídas salariales, de efectivos, recortes en precios de medicamentos y eliminación de inversiones, produciéndose unos ajustes asimétricos con unos altos costes diferidos en términos de debilitamiento del sistema sanitario (Rico y Blackey, 2012).

b) Políticas de ajuste en el empleo público: el capítulo I de los presupuestos de las Administraciones supone una parte importante en el gasto público (10,56\% del PIB en 2018) y es objeto de revisión cuando se abordan políticas de consolidación fiscal. Según el Banco de España (2017) entre los años 2012 y 2013 se produce una reducción de unos 350.000 efectivos públicos con una congelación efectiva de las tasas de reposición ligeramente superiores al 5 \% en los años 2012, 2013 y 2014.

c) Políticas de recentralización burocrática: se pusieron en marcha para un mayor control del gasto público y tuvieron como medida más significativa la reforma del artículo 135 de la Constitución Española y la ley orgánica 2/2012, de 27 de abril, de Estabilidad Presupuestaria y Sostenibilidad Financiera que establece unos principios rectores basados en la estabilidad y sostenibilidad y unos nuevos procedimientos para su aplicación efectiva que refuerzan los mecanismos de supervisión y control. Otra medida, de menor calado pero muy ilustrativa, es la derogación de la ley 28/2006, de 18 de julio, de Agencias estatales que suprime este mecanismo de descentralización en la gestión, novedoso en el panorama administrativo español.

d) Políticas de racionalización administrativa: la medida más destacada fue la reforma CORA (20122015) que sirvió para atajar duplicidades entre Administraciones, fortalecer servicios comunes, simplificar procedimientos y eliminar cargas administrativas, y «adelgazar» las Administraciones Públicas, todo ello con el resultado de 222 medidas para ahorrar 3.069 millones de euros (OPERA, 2016). Otra medida importante de este conjunto de políticas fue la ley $27 / 2013$, de 27 de diciembre, de Racionalización y Sostenibilidad de la Administración local cuyo propósito fue el de reordenación del gobierno local sobre la base de los principios de estabilidad presupuestaria y sostenibilidad financiera..

\section{Las políticas de gobierno abierto}

Surgen como respuesta a la desconfianza y con el objeto de reconectar a la ciudadanía con sus dirigentes políticos y con la Administración teniendo gran predicamento en el discurso de la reforma del sector público desde que en 2009 la Administración Obama dictara la primera orden ejecutiva sobre gobierno abierto.

Uno de los hitos en este conjunto de políticas fue la adhesión de España a la Alianza Internacional por el Gobierno Abierto (Open Government Partnership) que comprometió al país en la elaboración de Planes de Gobierno Abierto sujetos a evaluación por un Mecanismo Independiente de Revisión.

La ley 19/2013, de 9 de diciembre, de Transparencia, Acceso a la Información Pública y Buen Gobierno (LTBG) supuso una novedad al situar a España entre los países que imponen obligaciones en materia de transparencia (art. 5 y 8) y garantizar un nuevo derecho de acceso a la información pública (capítulo III). Pero es una ley que pronto se vio superada por la sucesiva aprobación de leyes autonómicas y ordenanzas municipales, muchas de ellas más ambiciosas que la ley estatal.

A la aprobación de la LTBG se han sumando otras medidas entre las que podríamos destacar las sucesivas leyes de participación electrónica aprobadas por las Comunidades Autónomas que sintetiza en su estudio Castel (2014), la creación de la Dirección General de Gobernanza Pública mediante el Real Decreto 769/2017 de 28 de julio, o el Foro de Gobierno Abierto creado en 2018 como primer espacio de naturaleza colaborativa que reúne a todas las Administraciones de nuestro país, el ámbito académico y la sociedad civil.

\section{LA COVID-19 COMO ACELERADOR DE LA DISRUPCIÓN EN EL ENTORNO}

La crisis ocasionada por el coronavirus SARS-CoV-2 está suponiendo un extraordinario desafío para la humanidad desde que a finales del año 2019 se conocen los primeros casos en la localidad china de Wuhan. Nos encontramos ante un fenómeno de un impacto excepcional a nivel sanitario, económico y social.

El impacto sanitario viene marcado por las cifras de contagiados y fallecidos, así como el enorme estrés que está suponiendo para los sistemas de salud y la comunidad científica internacional. Según datos de la 
GAPP. Nueva Época - N. ${ }^{2}$ 24, noviembre 2020 - ISSN: 1989-8991 - DOI: https://doi.org/10.24965/gapp.i24.10811 - [Págs. 6-23]

Organización Mundial de la Salud a fecha de 16 de septiembre de 2020 se contabilizaban 29.444 .198 casos confirmados y 931.321 muertos, siendo España el noveno país del mundo más afectado con un total de 603.167 casos y 30.004 fallecidos $^{7}$.

El impacto económico lo refleja la caída sin precedentes de la actividad por las restricciones impuestas para contener la transmisión del virus. Según Eurostat, la zona euro ha visto cómo su Producto Interior Bruto (PIB) ha experimentado un retroceso de un 12,1\% en el segundo trimestre con países como Alemania $(-10,1 \%)$, Italia $(-12,4 \%)$, Francia $(-13,8 \%)$ cayendo tasas históricas. España ha sido el país con mayor caída de toda la Unión Europea $(-18,5 \%)$, casi 6 puntos porcentuales por encima de la media de los países de la Unión. Esta situación tiene una profunda huella social derivada del aumento del desempleo y el ensanchamiento de las brechas de desigualdad generadas durante la Gran Recesión.

Este fenómeno de alto impacto es un factor de contexto de gran relevancia para las políticas públicas. Siguiendo la clasificación de Mintzberg (1984) podríamos indicar que la crisis de la COVID-19 da lugar a un entorno altamente disruptivo atendiendo a los diferentes atributos de muy volátil, incierto, extremadamente complejo y ambiguo ${ }^{8}$.

TABLA 3. LA COVID-19 COMO FACTOR DE UN ENTORNO ALTAMENTE DISRUPTIVO

\begin{tabular}{ll}
\hline \multicolumn{1}{c}{ Atributo } & \multicolumn{1}{c}{ Efectos de la COVID-19 } \\
\hline Volatilidad & $\begin{array}{l}\text { El contexto cambia continuamente en periodos muy cortos. Como ejemplo, las decisiones sobre } \\
\text { restringir la movilidad anunciadas con poca antelación para que sean efectivas. }\end{array}$ \\
\hline Incertidumbre & $\begin{array}{l}\text { No sabemos cuándo y cómo terminará. Todavía es demasiado pronto para predecir cómo cambiará } \\
\text { nuestra sociedad. }\end{array}$ \\
\hline Ambigüedad & $\begin{array}{l}\text { La difícil manifestación del problema en todas sus dimensiones. Extraordinarios interrogantes cien- } \\
\text { tíicos que impiden definir con nitidez los caminos de la recuperación. }\end{array}$ \\
\hline Complejidad & $\begin{array}{l}\text { No existe una solución. Dificultad para cuantificar la efectividad de las medidas adoptadas. La retor- } \\
\text { cida relación entre salud y economía. }\end{array}$ \\
\hline
\end{tabular}

Fuente: Elaboración propia.

\section{FASES DE LA CRISIS DE LA COVID-19 Y DECISIONES PÚBLICAS MÁS RELEVANTES}

No es éste el lugar para pararnos en detalle sobre lo que ha supuesto en términos sanitarios, económicos y sociales la crisis de la COVID-19 que está asolando a España y al mundo. Nuestro interés tiene que ver con el impacto que dicha crisis ha tenido en las instituciones y en las políticas para su reforma.

El análisis que llevamos a cabo abarca los seis meses que tienen lugar a partir de la declaración del estado de alarma en España el pasado 14 de marzo de 2020. Durante este periodo establecemos tres fases en las que detallaremos las medidas más importantes desde el punto de vista de su impacto en la Administración.

\section{a) Primera fase. Confinamiento}

Corresponde al periodo entre el 14 de marzo y finales de abril en el que se produce un crecimiento epidémico exponencial que provoca el colapso del sistema sanitario. En muy poco tiempo se alcanzan cifras de

7 El número total de fallecidos en España es un dato controvertido ya que existen importantes discrepancias entre la cifra oficial de fallecidos (Ministerio de Sanidad) y los anotados en los registros civiles recogidos por el Sistema de Monitorización de la Mortalidad (MoMo). Véase: https://elpais.com/sociedad/2020/05/27/actualidad/1590570927_371193.html.

8 Algunos incluso la califican de cisne negro (Taleb, 2012) al ser un suceso altamente improbable pero que habiéndose dado provoca efectos extraordinarios. 
GAPP. Nueva Época - N. ${ }^{2}$ 24, noviembre 2020 - ISSN: 1989-8991 - DOI: https://doi.org/10.24965/gapp.i24.10811 - [Págs. 6-23]

casi 10.000 contagiados y 1.000 muertos diarios lo que obliga a la adopción de las medidas excepcionales más drásticas.

Se aprueba el Real Decreto 463/2020, de 14 de marzo, por el que se declara el estado de alarma para la gestión de la situación de crisis sanitaria ocasionada por el COVID-19 y, tras él, una catarata normativa que tiene por objeto ir concretando medidas.

TABla 4. Medidas e impactos más ReleVANtes Sobre la Administración duRANTe La fase de confinamiento

\begin{tabular}{ll}
\hline Contenido & Referencia \\
\hline & Gobernanza \\
\hline
\end{tabular}

Creación de un mando único que otorga poderes especiales a los minis- RD 463/2020 de 14 de marzo, art. 4.2. tros (dictar órdenes, resoluciones, disposiciones e instrucciones interpretativas).

El Ministerio de Sanidad, núcleo del mando único, centraliza decisiones, no sólo de coordinación y política básica sanitaria, sino de distribución de RD 463/2020 de 14 de marzo, art. 12 y 13. medios técnicos y personales.

Conferencia de presidentes semanal.

\section{Servicios públicos}

Cierre de la actividad presencial en el sector público, incluyendo áreas sectoriales como educación y justicia, en la que incluso se cierran servicios digitales para la presentación de escritos y actos de comunicación.

Paso al teletrabajo de los empleados públicos.

RD 463/2020 de 14 de marzo, art. 9 Acuerdo de la Comisión Permanente del Consejo General del Poder Judicial, de 11 de marzo.

Subsidios de desempleo excepcionales.

RDL 11/2020 de 31 de marzo.

Medidas de flexibilización de los mecanismos de ajuste temporal (ERTE). RDL 8/2020 de 7 de marzo.

\section{Digitalización y procedimientos}

Suspensión de los plazos administrativos y procesales, lo que de facto supone la paralización administrativa.

Identificación por videoconferencia para la expedición de certificados RDL 11/2020 de 31 de marzo. electrónicos cualificados.

Pago de deudas tributarias con tarjetas de crédito y débito mediante sistema Cl@vePIN.
RD 463/2020 de 14 de marzo,

disposiciones adicionales 2. ${ }^{a}, 3 .^{a}$ y $4 .^{a}$.

Resolución de 15 de abril de la Dirección General de la Agencia Estatal de la Administración Tributaria.

Meijer y Webster, 2020.

Orden SND/297/2020 de 27 de marzo. Criado, Guevara-Gómez y Villodre, 2020.

Orden SND/233/2020 de 15 de marzo.

Fuente: Elaboración propia.

\section{b) Segunda fase. Desescalada}

La segunda fase corresponde al periodo entre finales de abril y primeros de junio en el que se produce un descenso significativo en el número de contagios y fallecidos lo que permite ir tomando medidas de alivio en las restricciones adoptadas. 
GAPP. Nueva Época - N. ${ }^{2}$ 24, noviembre 2020 - ISSN: 1989-8991 - DOI: https://doi.org/10.24965/gapp.i24.10811 - [Págs. 6-23]

El llamado Plan para la Transición hacia una Nueva Normalidad, aprobado el 28 de abril, establece una batería de decisiones para la recuperación gradual de la actividad en distintas fases: la fase 0 o de preparación a la desescalada, la fase 1 o inicial, la fase 2 o intermedia, la fase 3 o avanzada y la nueva normalidad.

Se aprueban sucesivas órdenes ministeriales para regular los aspectos de este plan de desescalada. Las más significativas son las que regulan las diferentes fases: Orden SND/399/2020 de 9 de mayo (fase 1), Orden SND/414/2020 de 16 de mayo (fase 2) y Orden SND/458/2020 de 30 de mayo (fase 3) a las que acompañan de nuevo otras muchas medidas de las que las más significativas a los efectos de este estudio son las siguientes:

TABla 5. Medidas e impactos más ReleVAntes Sobre la Administración duRANTE La fase de desescalada

\begin{tabular}{|c|c|}
\hline Contenido & Referencia \\
\hline \multicolumn{2}{|c|}{ Gobernanza } \\
\hline $\begin{array}{l}\text { Mayor participación de las Comunidades Autónomas en } \\
\text { toma de decisiones sanitarias. }\end{array}$ & Orden SND/387/2020 de 3 de mayo. \\
\hline \multicolumn{2}{|l|}{ Conferencia de presidentes semanal. } \\
\hline Refuerzo de la coordinación en salud pública. & $\begin{array}{l}\text { Orden SND/404/2020 de } 11 \text { de mayo y RDL } 21 / 2020 \\
\text { de } 9 \text { de junio. }\end{array}$ \\
\hline \multicolumn{2}{|c|}{ Servicios públicos } \\
\hline $\begin{array}{l}\text { Reincorporación programada del personal de la } \\
\text { Administración de Justicia. }\end{array}$ & $\begin{array}{l}\text { Orden JUS/394/2020 de } 8 \text { de mayo, Orden } \\
\text { JUS/430/2020 de } 22 \text { de mayo y Orden JUS/504/2020 } \\
\text { de } 5 \text { de junio. }\end{array}$ \\
\hline Refuerzo de la vigilancia epidemiológica. & $\begin{array}{l}\text { Orden SND/404/2020 de } 11 \text { de mayo y RDL 21/2020 } \\
\text { de } 9 \text { de junio. }\end{array}$ \\
\hline $\begin{array}{l}\text { Medidas para garantizar la capacidad del sistema } \\
\text { sanitario (recursos humanos y planes de contingencia). }\end{array}$ & RDL 21/2020 de 9 de junio. \\
\hline $\begin{array}{l}\text { Acuerdos de la Conferencia Sectorial de Educación (inicio } \\
\text { del curso 20-21). }\end{array}$ & Orden EFP/561/2020 de 20 de junio. \\
\hline Ingreso mínimo vital. & RDL 20/2020 de 29 de mayo. \\
\hline \multicolumn{2}{|c|}{ Digitalización y procedimientos } \\
\hline Gestión de datos sobre vigilancia epidemiológica. & Orden SND/404/2020, de 11 de mayo. \\
\hline $\begin{array}{l}\text { Actos procesales mediante presencia telemática y } \\
\text { agilización actuaciones. }\end{array}$ & RDL 16/2020 de 28 de abril. \\
\hline $\begin{array}{l}\text { Procedimientos administrativos mediante clave } \\
\text { concertada. }\end{array}$ & $\begin{array}{l}\text { Resolución de } 29 \text { de abril de } 2020 \text { de la Secretaría } \\
\text { General de Administración Digital. }\end{array}$ \\
\hline
\end{tabular}

Fuente: Elaboración propia.

\section{c) Tercera fase. Nueva normalidad y rebrotes}

La tercera fase corresponde al periodo comprendido entre los meses de junio y mediados de septiembre en el que tras una fase inicial de contención de la epidemia se empiezan a producir rebrotes que acaban por desbordar el control de las autoridades sanitarias ${ }^{9}$.

Se aprueba el llamado decreto de la nueva normalidad RDL 21/2020, de 9 de junio, de medidas urgentes de prevención, contención y coordinación para hacer frente a la crisis sanitaria ocasionada por el COVID-19 y se comienzan a poner en marcha nuevos planes y estrategias para la reactivación económico y social.

9 A mediados de septiembre la situación en Madrid obliga al gobierno regional a adoptar medidas restrictivas el 18 de septiembre mediante la Orden $1177 / 2020$. 
GAPP. Nueva Época - N. ${ }^{2}$ 24, noviembre 2020 - ISSN: 1989-8991 - DOI: https://doi.org/10.24965/gapp.i24.10811 - [Págs. 6-23]

TABla 6. Medidas e impactos más ReleVANTES Sobre LA AdMinistración duRANTE LA fase de nUEVA NORMALIDAD Y REBROTES

\begin{tabular}{|c|c|}
\hline Contenido & Referencia \\
\hline \multicolumn{2}{|l|}{ Gobernanza } \\
\hline $\begin{array}{l}\text { Cogobernanza del Ministerio de Sanidad con las Comunidades Au- } \\
\text { tónomas para toma de decisiones sobre desescalada. }\end{array}$ & RDL 21/2020 de 9 de junio. \\
\hline \multicolumn{2}{|l|}{ Refuerzo Consejo Interterritorial del Sistema Nacional de Salud. } \\
\hline Creación de Secretaría de Estado de Sanidad. & RD $722 / 2020$ de 31 de julio. \\
\hline \multicolumn{2}{|l|}{ Conferencia de presidentes mensual. } \\
\hline \multicolumn{2}{|l|}{ Servicios públicos } \\
\hline $\begin{array}{l}\text { Acuerdo en sanidad y salud pública para la reconstrucción aproba- } \\
\text { do por el Congreso de los Diputados. }\end{array}$ & $\begin{array}{l}\text { Conclusiones para la Reconstrucción Social y } \\
\text { Económica - Sanidad y Salud Pública. }\end{array}$ \\
\hline $\begin{array}{l}\text { Medidas para garantizar la capacidad del sistema sanitario (recur- } \\
\text { sos humanos y planes de contingencia). }\end{array}$ & RDL 21/2020 de 9 de junio. \\
\hline $\begin{array}{l}\text { Acuerdo del Consejo Europeo para el Plan de Recuperación eu- } \\
\text { ropeo. }\end{array}$ & $\begin{array}{l}\text { Conclusiones de Consejo Europeo } 17 \text { a } 21 \\
\text { julio. }\end{array}$ \\
\hline \multicolumn{2}{|c|}{ Digitalización y procedimientos } \\
\hline \multicolumn{2}{|l|}{ Estrategia España Digital 2025.} \\
\hline Teletrabajo en la Administración General del Estado. & $\begin{array}{l}\text { Resolución del Secretario de Estado de } \\
\text { Política Territorial y Función Pública de } 17 \text { de } \\
\text { junio. } \\
\text { Acuerdo sobre teletrabajo en la Mesa General } \\
\text { de Negociación de las Administraciones } \\
\text { Públicas de } 21 \text { de septiembre. }\end{array}$ \\
\hline \multicolumn{2}{|l|}{ Puesta en marcha de RADAR COVID-19. } \\
\hline $\begin{array}{l}\text { Creación de la Dirección General de Salud Digital y Sistemas de } \\
\text { Información para el Sistema Nacional de Salud. }\end{array}$ & RD 735/2020 de 4 de agosto. \\
\hline Creación de la Oficina del Dato. & Orden ETD 803/2020 de 31 de julio. \\
\hline
\end{tabular}

Fuente: Elaboración propia.

\section{LA ADMINISTRACIÓN TRAS EL CORONABREAK. DISCUSIÓN Y PROPUESTAS}

En el presente estudio hemos identificado una serie de políticas de reforma administrativa que se venían aplicando antes de la llegada de la crisis ocasionada por la COVID-19: digitalización, ajuste administrativo y gobierno abierto. Las tres condicionadas por un factor de contexto claro, la Gran Recesión.

Por otra parte, hemos visto cómo la COVID-19 ha planteado un entorno disruptivo con impactos significativos sobre las tres variables del funcionamiento de la Administración pública que se han analizado: gobernanza, servicios públicos y digitalización y procedimientos.

La discusión se centra en analizar cómo será esta incidencia en las políticas que ya se venían desarrollando, además de identificar nuevas políticas que pudieran ponerse en marcha en el futuro más inmediato.

\section{a) La aceleración de las políticas de digitalización del sector público}

Aunque las políticas de digitalización venían desarrollándose en la Administración desde hace dos décadas la crisis de la COVID-19 ha puesto de manifiesto que estas políticas no habían alcanzado el resultado deseado. Como muestra tres ejemplos: 
- La tramitación administrativa y judicial ha tenido que ser paralizada haciendo necesarias las disposiciones $2 .^{a}$ y $3 .^{a}$ del RDL 463/2020 que declaran la suspensión de plazos procesales y administrativos.

- El teletrabajo, aunque se intentó como experiencia mediante Orden APU/1982/2006, de 21 de junio, por la que se promueve la implantación de programas piloto en los departamentos ministeriales, pero hasta la llegada de la crisis no ha sido una realidad.

- La brecha digital ha obstaculizado el día a día de aquellos ciudadanos que, careciendo de conocimientos y herramientas básicos, se han visto en la necesidad de hacer trámites electrónicos para asuntos tan esenciales como pedir una prestación.

Las medidas tomadas durante los primeros seis meses de la crisis de la COVID-19 invitan a pensar en una aceleración de las políticas de digitalización en la Administración en consonancia con la tendencia del sector privado ${ }^{10}$. La recién aprobada estrategia España Digital 2025 y las medidas tomadas durante este periodo para mejorar la identidad digital, la consideración de nuevos trámites telemáticos, la simplificación de procedimientos, la regulación del teletrabajo o el uso de aplicaciones para atajar importantes problemas públicos son un ejemplo que invita a pensar que se consolidará esta tendencia y señalan líneas de actuación que pueden adquirir importancia en los próximos años.

En primer lugar, es preciso reducir la brecha digital y aumentar la accesibilidad de los servicios, entendida como la capacidad real y efectiva de los ciudadanos para usar aquellos que se ponen a su disposición. La simplificación de los procesos de identidad y firma es un objetivo necesario para que el uso de los servicios públicos digitales sea universal y que en posibles crisis como la de la COVID-19 no queden personas en situación de indefensión y privación de derechos. O actuaciones específicas sobre servicios esenciales, como la educación, dotando de equipamiento y competencias digitales a profesores y familias.

En segundo lugar, dinamizar el funcionamiento administrativo para dotar a los trámites de mayor agilidad. La inteligencia artificial puede contribuir a automatizar procesos, ofrecer una atención ciudadana más eficiente, ser más eficaces en combatir problemas colectivos o ayudar a la toma de decisiones. En áreas como la Seguridad Social, estratégica a nivel prestacional, se viene ya planteando el uso de robots para automatizar tareas repetitivas o detectar fraude. Además, durante los seis meses de pandemia se han puesto en marcha aplicaciones (Asistencia COVID-19, Hispabot COVID-19, Radar COVID-19) y se han realizado estudios de movilidad de la población para conocer la evolución. Sin embargo, su aplicación está condicionada por dos factores relevantes: por un lado, la madurez de las soluciones tecnológicas, su aplicabilidad, fiabilidad y escalabilidad en ámbitos como la Administración en los que las posibilidades de experimentar están muy condicionadas; por otro, lo delicado del sujeto de aplicación (por los bienes jurídicos que maneja) requiere de un marco legal y ético que garantice la seguridad jurídica, los derechos de ciudadanía y el interés general.

En tercer lugar, la gestión del dato y la interoperabilidad, especialmente en servicios esenciales muy descentralizados como los sanitarios. La primera es clave para permitir el desarrollo de la inteligencia artificial, desplegar una gestión más transparente y tomar decisiones basadas en la evidencia. La no interoperabilidad puede suponer, como hemos visto en esta crisis del COVID-19, un obstáculo en la prestación de servicios y la gestión de la información. Para ello es preciso desaprender y abordar el despliegue de la Administración digital desde criterios de racionalidad y eficacia. Potenciar los servicios comunes, la reutilización de soluciones, y las ventanillas únicas digitales son propuestas clave en esta estrategia. Pasos en esta dirección se han dado durante estos meses de pandemia con la creación de la Oficina del Dato y la puesta en marcha de la Dirección General de Salud Digital y Sistemas de Información para el Sistema Nacional de Salud que gobierne la estrategia de transformación digital en un sector como el sanitario donde la interoperabilidad es imprescindible.

El desafío es que este impulso de transformación técnico-jurídica no agrave los desajustes entre garantías y usabilidad y sea capaz de conciliar atribución competencial y eficacia tecnológica. Aunque la digitalización indudablemente debe desplegarse en concordancia con los principios del Estado de derecho y el reparto competencial en un Estado compuesto como el nuestro, de cara al futuro debe vigilarse que exista

10 El reciente I Barómetro COVID-19 y Marketing Good Rebels subraya que la crisis del COVID-19 será un formidable catalizador de la transformación digital de las empresas. Se habla además de un importante volumen de fondos, próximo a los 20.000 millones de euros, que llegarán de Europa parte para la modernización tecnológica de la Administración. 
un equilibrio eficaz que no dificulte el acceso de las tecnologías a la ciudadanía y se preserven pilares básicos como la privacidad y la seguridad, exigencias que se han reproducido con fuerza durante los meses de confinamiento a la hora de plantearse soluciones técnicas para rastrear el virus a partir de la movilidad y los contactos de cada individuo ${ }^{11}$.

\section{b) De las políticas de ajuste administrativo a políticas de fortalecimiento institucional}

Veíamos anteriormente cómo tras la Gran Recesión (2008-2013) las políticas de ajuste administrativo han impactado sobre los servicios esenciales, el empleo público y la organización administrativa. La crisis de la COVID-19 ha puesto de manifiesto importantes debilidades en servicios públicos (nuevos o ya existentes, como los sanitarios o las prestaciones sociales) y desafíos en gobernanza y capacidad de gestión.

Las medidas adoptadas durante estos primeros seis meses de pandemia apuntan hacia una necesidad de fortalecimiento que podría consolidarse en los próximos años. En primer lugar, la crisis sanitaria ha puesto de manifiesto la necesidad de actuar sobre los servicios esenciales. La sanidad es una de las áreas de reforma más evidente: se trata de corregir las debilidades mostradas y dotar al sistema de nuevas capacidades de cara a futuras pandemias. El acuerdo suscrito por las fuerzas parlamentarias para la reconstrucción del Sistema Nacional de Salud es un punto de partida que muestra algunas de las actuaciones más inmediatas: una política de recursos humanos del Sistema Nacional de Salud con objetivos a largo plazo, un impulso a la Atención Primaria y Comunitaria, así como una Estrategia de Transformación Digital para la Sanidad. Por otra parte, el sistema administrativo ha fallado a la hora de poner en marcha nuevos servicios: el colapso en la tramitación de los expedientes de regulación temporal de empleo (ERTE) o del recién creado Ingreso Mínimo Vital ponen en evidencia la necesidad de reforzar las capacidades de las Administraciones públicas en determinados sectores (por ejemplo, el de la política social), simplificar procedimientos y ahondar en la digitalización.

La crisis de la COVID-19 también nos ha mostrado como ante escenarios de extraordinaria incertidumbre se requieren buenas dosis de gobernanza exploratoria (Longo, 2019) que permita incorporar sistemas de detección, diagnóstico, anticipación e innovación. Nuestras Administraciones debieran disponer de la capacidad para encontrar respuestas y soluciones en cortos espacios de tiempo a problemas a los que nunca se habrían enfrentado. Serán necesarias políticas de simplificación de procedimientos -rediseñándolos y en algunas ocasiones reinventándolos- poniendo el foco de las garantías en aquellos trámites donde tengan más efecto desde una perspectiva coste-beneficio y en aquellos servicios más relevantes para empresas y ciudadanos. Los esfuerzos deben ir encaminados a eliminar redundancias, abstenerse de pedir información que no sea la estrictamente necesaria o que ya esté en posesión de la Administración o establecer controles ex post por proceso y no por trámite.

El caso del Ingreso Mínimo Vital es significativo. Con amplio consenso parlamentario, su puesta en marcha como infraestructura social básica del Estado del bienestar, no ha estado exenta de críticas y dificultades. La lentitud de la tramitación -hasta el 7 de agosto solo se habían aprobado 3.966 solicitudes de un volumen total de $510.000^{12}$ - ha forzado una revisión del procedimiento eliminando requisitos previos y fomentando el cruce masivo de datos ${ }^{13}$.

Otro ámbito que aventuramos será objeto de las políticas de reforma será el de la gobernanza y los sistemas de decisión en el marco del Estado de las Autonomías, especialmente en el ámbito sanitario. Durante los seis meses de pandemia se ha pasado de un mando único central como respuesta excepcional a una situación excepcional a una llamada cogobernanza en la que las Comunidades Autónomas han retomado sus competencias sanitarias para combatir la pandemia en solitario con la coordinación de una recién creada Secretaría de Estado de Sanidad y el reforzado Consejo Interterritorial del Sistema Nacional de Salud, solución que no ha ofrecido buenos resultados a tenor del fuerte rebrote de la pandemia ${ }^{14}$. El

11 Véase: https://hayderecho.expansion.com/2020/04/10/el-interes-publico-de-los-datos-personales-en-tiempos-del-covid-19/.

12 Véase: https://www.elconfidencial.com/espana/2020-08-19/imv-hogares-espana-pobreza-ingreso-minimo-vital_2718159/.

13 Véase: https://revista.seg-social.es/2020/09/22/el-ministerio-de-inclusion-mejora-los-procedimientos-del-ingreso-minimo-vital/.

14 Ante el incremento descontrolado de los contagios en la Comunidad de Madrid, el 21-09-20 el presidente del Gobierno, Pedro Sánchez, y la presidenta de la Comunidad de Madrid, Isabel Díaz Ayuso, se reúnen para crear una nueva estructura de coordinación que estreche el trabajo conjunto para combatir la pandemia en la región. Véase: https://www.elmundo.es/madrid/2020/09/21/5f688979 21efa0f7578b467e.html. 
dictamen aprobado por la Comisión de Reconstrucción del Congreso de los Diputados recomienda reforzar la gobernanza del sistema sanitario español y plantea medidas como la creación de un Centro Estatal Salud Pública que impulse una colaboración más estrecha entre las autoridades competentes y plantee políticas conjuntas más eficaces.

El mando único ha puesto de manifiesto importantes carencias de nuestra Administración en la gestión y ejecución, por ejemplo, en áreas como la compra y suministro de material sanitario -incluyendo la negociación e identificación de proveedores, la gestión logística, la colaboración público-privada- o la toma de decisiones sobre operativa sanitaria. Apunta Dorrego (2020: 25-26) como causas la «notoria ausencia de instrumenta regna imprescindibles para asumir tales poderes: estructura administrativa robusta, dotada de altos funcionarios especializados y bien preparados, con engranajes de dirección y coordinación eficientes». Limitaciones que se pueden hacen aún más evidentes a la hora de gestionar los fondos para la reconstrucción aprobados en el Consejo Europeo de mediados de julio.

Por otra parte, las Administraciones públicas se han visto necesitadas de recursos en las áreas más críticas ${ }^{15}$ en detrimento de otras que debido al desajuste ocasionado por la pandemia han quedado paradas, tendencia que previsiblemente será más acusada en los próximos años.

Adquirirá una importancia capital la reforma del empleo público que flexibilice la gestión permitiendo una distribución de efectivos más eficiente y permita el fortalecimiento de las capacidades de dirección, planificación y gestión en sectores estratégicos a través de una función directiva profesional por la que autores como Arenilla y Delgado (2019) o Longo (2008) vienen apostando en los últimos años.

\section{c) Las políticas de gobierno abierto en la encrucijada}

Las políticas de gobierno abierto venían adquiriendo gran predicamento en los años anteriores al coronabreak. Sin embargo, la crisis sanitaria no ha supuesto un impulso a este conjunto de políticas, siendo escasas las medidas adoptadas en favor de la transparencia, la participación o la rendición de cuentas en estos seis meses que llevamos de pandemia.

Por el contrario, la transparencia se ha visto lastrada por la excepcionalidad. No ha habido ninguna medida para reforzar la publicidad activa, habiéndose incluso orillado ${ }^{16}$ con la excepción de algunas instituciones que han hecho públicos datos como los gastos incurridos para hacer frente a la pandemia ${ }^{17}$. El derecho de acceso a la información se ha bloqueado en todas las Administraciones al amparo de la suspensión de plazos administrativos decretado por el RD 463/2020.

La gestión de los datos se ha visto penalizada por las deficiencias en interoperabilidad y digitalización de los procesos o los desencuentros en cuanto a las fuentes y su interpretación, impidiéndose políticas y tomas de decisiones basadas en la evidencia ${ }^{18}$.

Por el contrario, la actividad de comunicación durante todos estos meses (especialmente en la fase de confinamiento) ha sido intensa. Las campañas en redes sociales fueron recurrentes para movilizar a la ciudadanía, sensibilizarla y recabar su colaboración (Criado, Guevara-Gómez y Villodre, 2020). Las comparecencias de los dirigentes (políticos y técnicos) también han sido numerosas. Sin embargo, la rendición de cuentas ha esquivado una evaluación y análisis independiente de las decisiones adoptadas. ${ }^{19}$.

La paradoja es que este abandono de la transparencia y la rendición de cuentas ocurre cuando la necesidad de saber es más apremiante que nunca. De ahí los interrogantes sobre cómo van a evolucionar las políticas de gobierno abierto si en un escenario como el de la COVID-19, en el que la demanda de transparencia e información es máxima, los mecanismos establecidos no han acabado de funcionar correctamente.

15 El propio ministro José Luis Escrivá reconoce que la Seguridad Social sufre falta de personal. Véase: https://www.elconfidencial. com/economia/2020-08-06/escriva-seguridad-social-falta-personal_2707275/.

16 Véase: https://www.abc.es/espana/abci-comunidad-madrid-tambien-limita-servicios-transparencia-durante-estado-alarma-202004280208 noticia.html.

17 Véase: https://transparencia.madrid.es/portales/transparencia/es/Portada/Informacion-Covid-19/Actuaciones-presupuestariasCOVID-19/?vgnextfmt=default\&vgnextoid=32b760ff77781710VgnVCM2000001f4a900aRCRD\&vgnextchannel=e8d97cbbafd71710VgnV CM2000001f4a900aRCRD.

18 Véase: $h$ ttp://agendapublica.elpais.com/por-un-sector-publico-capaz-de-liderar-la-recuperacion/.

19 En una carta publicada a primeros de agosto en The Lancet, una veintena de expertos en salud pública piden al gobierno español una evaluación independiente e imparcial sobre la gestión de la pandemia, con el ánimo de aprender sobre lo sucedido y evitar errores de cara a nuevas olas de contagios. 
Cabe pensar sin embargo que ante un escenario post COVID-19 la transparencia no sólo no debería verse resentida, sino que debiera reforzarse dejando atrás la retórica del discurso político y alcanzando una «transparencia real que cristalice en obligaciones concretas que garanticen esa redistribución de derechos y responsabilidades entre sociedad y gobierno» como reclama Cotino (2013: 65-67).

Se trataría de profundizar en la transparencia y el buen gobierno a partir de tres atributos: la accesibilidad -que la información sea fácilmente accesible a cualquier persona, en cualquier momento y en cualquier lugar; la receptividad- desde el gobierno y/o la Administración; y la equidad, para equilibrar la brecha entre actores con mayor y menor capacidad de acceso. Obligaciones que podrían plasmarse en algunos de los retos jurídicos aún pendientes: la aprobación del reglamento de la $\mathrm{LTBG}^{20}$, el inacabado debate sobre si el acceso a la información ha de ser un derecho fundamental y la mayor autonomía orgánica, funcional y en medios del Consejo de Transparencia y Buen Gobierno.

\section{CONCLUSIONES}

Doscientos años después del nacimiento de la Administración pública han sido muchos los hitos históricos que han impactado en los diferentes subsistemas de lo público. Guerras mundiales, crisis económicas, globalización, revolución digital son algunos de los fenómenos tras los diversos paradigmas de transformación del sector público. De las reformas que se han sucedido, tres han sido las que han protagonizado las primeras dos décadas de este siglo: las políticas de digitalización, las de ajuste administrativo y las de gobierno abierto.

La crisis de la COVID-19 está suponiendo, a todos los niveles, un shock considerable y representa un factor de entorno de características altamente disruptivas por su alto impacto y duración sostenida que agita una elevada incertidumbre y complejidad.

Durante los primeros seis meses de pandemia se han sucedido diferentes fases de confinamiento, desescalada, nueva normalidad y rebrotes hasta alcanzar una segunda ola epidémica. En este periodo las instituciones han tenido un papel central y han tomado numerosas medidas para combatir este problema. Una parte de ellas han mostrado las dificultades administrativas para responder con eficacia a contextos de esta naturaleza, poniendo en evidencia que las políticas de reforma llevadas a cabo hasta la fecha no han sido lo suficientemente eficaces: la inacabada digitalización, la fragilidad del sistema sanitario, los procedimientos obsoletos o mecanismos de gobernanza no asentados son algunos ejemplos.

Otra parte han sido medidas con impactos sobre el funcionamiento administrativo que dan pistas sobre el rumbo que pudieran tomar las nuevas políticas de reforma. Analizándolas y contrastándolas con las políticas que se venían desarrollando parece verosímil concluir que, en momentos en los que la crisis del COVID-19 ha acelerado los atributos de volatilidad, incertidumbre, complejidad o ambigüedad del entorno y cuando el valor generado por lo público es más relevante que nunca al tener que responder de manera eficaz ante riesgos complejos, sea necesario dar un salto adelante en políticas de reforma en una doble dirección: la transformación y el fortalecimiento.

Las políticas de transformación tienen que ver con acelerar las políticas de digitalización que se venían desarrollando hasta el momento. Se trataría de aprovechar dos palancas fundamentales -tecnología e innovación- para que la Administración pueda avanzar en el uso de la tecnología y replantearse muchos de sus procedimientos. La introducción de la inteligencia artificial, la gestión por datos, el impulso de sistemas de gestión común que facilite la interoperabilidad o la universalización de la inteligencia artificial son algunas propuestas.

Por otro lado, la crisis de la COVID-19 nos ha mostrado que en situaciones límite el aparato administrativo ha de responder y para ello es preciso ampliar su capacidad y agilidad para tramitar y poner en marcha servicios de alta accesibilidad, tomando decisiones de manera más precisa, con más transparencia y distribuyendo los recursos de manera más efectiva. Las políticas de fortalecimiento han de permitir avanzar en estos objetivos trabajando en una doble dirección: el refuerzo de los servicios públicos esenciales, especialmente los sanitarios, y los mecanismos de cooperación y gobernanza; por otra parte, una reforma del empleo público para que las instituciones dispongan del músculo necesario en términos de capacidades, profesionalización y talento.

20 Seis años después de la publicación de la ley 19/2013 el reglamento está aún en forma de borrador de Real Decreto. La tramitación de esta norma puede consultarse en: https://transparencia.gob.es/transparencia/transparencia_Home/index/ParticipacionCiudadana/ParticipacionProyectosNormativos/proyectoRDTransparencia.html. 


\section{REFERENCIAS BIBLIOGRÁFICAS}

ACEMOGLU, D.; ROBINSON, J. (2012): Why nations fail. The origins of power, prosperity and poverty. Londres: Profile Books.

ARENILLA, M.; DELGADO, D. (2019): “Una nueva función pública que fortalezca la confianza en las instituciones públicas", en Revista Vasca de Gestión de Personas y Organizaciones Públicas, núm. 16, págs. 36-53.

Ayuso y Sánchez entierran la confrontación y crean grupos de trabajo conjuntos para frenar la COVID-19 en Madrid (2020), en El Mundo. Fecha de consulta: 22-09-20. URL: https://www.elmundo.es/madrid/2020/09/21/5f68897921 efaOf7578b467e.html.

BANDRÉS, E.; GONZÁLEZ, R. (2015): "La reducción del gasto sanitario en España durante la crisis", en Cuadernos de Información Económica, núm. 248, págs. 38-48.

Barómetro especial mayo (2020). Madrid: Centro de Investigaciones Sociológicas. Fecha de consulta: 02-08-20. URL: http://datos.cis.es/pdf/Es3281marMT_A.pdf.

BARZELAY, M. (1998): Atravesando la burocracia, una nueva perspectiva de la Administración pública. México D. F.: Fondo de Cultura económica.

BERMAN, P. (1980): “Thinking about Programmed and Adaptive Implementation: matching strategies to situations", en INGREM, H. y MANN, D. (coords.): Why policies succeed or fail (205-227). Beverly Hills: Sage Publications.

BROWN, D. (2005): "Electronic government and public administration", en International Review of Administrative Sciences, vol. 71, núm. 2, págs. 241-254. DOI: https://doi.org/10.1177/0020852305053883.

CASTEL, S. (2014): "Marco normativo e institucional del nuevo derecho de participación y las TIC: análisis desde las experiencias autonómicas", en Revista de Internet, Derecho y Política, núm. 19, págs. 48-61. DOI: https://doi. org/10.7238/idp.v0i19.2332.

Conclusiones de la Comisión para la Reconstrucción Social y Económica - Sanidad y Salud Pública (2020). Madrid: Congreso de los Diputados. Fecha de consulta: 02-09-2020. URL: http://www.congreso.es/docu/comisiones/ reconstruccion/153_1_Aprobacion_Pleno.pdf.

Conclusiones sobre el Consejo Europeo 17 a 21 julio 2020 (2020). Bruselas: Consejo Europeo. Fecha de consulta: 0409-2020. URL: https://www.consilium.europa.eu/media/45124/210720-euco-final-conclusions-es.pdf.

CORTÉS, O. (2019): Las redes sociales en la Administración General del Estado. Factores jurídicos e institucionales. Tesis doctoral. A Coruña: Universidad de A Coruña. Fecha de consulta: 02-07-2020. URL: https://ruc.udc.es/ dspace/bitstream/handle/2183/24407/CortesAbad_Oscar_TD_2019.pdf.

COTINO, L. (2013): "Derecho y "Gobierno Abierto». La regulación de la transparencia y la participación y su ejercicio a través del uso de las nuevas tecnologías y las redes sociales por las Administraciones públicas. Propuestas concretas", en Monografías de la Revista Aragonesa de Administración Pública, núm. extra 14, págs. 51-92.

CRIADO, I.; ROJAS, F. (2015a): "El impacto de las redes sociales digitales en las Administraciones locales. Mitos y realidades en el caso español", en Revista de Internet, Derecho y Política, núm. 20, págs. 25-42. DOI: https://doi. org/10.7238/idp.v0i20.2581.

CRIADO, I.; ROJAS, F. (2015b): "Estrategias y realidades en la difusión de las redes sociales en las administraciones públicas. Análisis desde una perspectiva institucionalista", en Innovar, vol. 25, núm. 57, págs. 45-62. DOI: https:// doi.org/10.15446/innovar.v25n57.50326.

CRIADO, I.; GUEVARA-GÓMEZ, A.; VILLODRE, J. (2020): "Using collaborative technologies and social media to engage citizens and governments during the COVID-19 Crisis. The case of Spain", en Digital Government: Research and Practice, september 2020, núm. 30. DOI: https://doi.org/10.1145/3416089.

DANS, E. (2010): “La empresa y la web 2.0", en Deusto Marketing y Ventas, núm. 80, págs. 36-43.

DORREGO, A. (2020): "Los servicios públicos en la de pandemia”, en OtroSí, págs. 24-27.

ECHEBARRÍA, K. (1998): "Los dilemas del diseño organizativo en la gestión pública", en LONGO, F. e YSA, T. (eds.): Los nuevos escenarios de la gestión pública en el siglo XXI, págs. 85-114. Barcelona: Generalitat de Catalunya.

Ejecución presupuestaria del programa 231.99. Actuaciones extraordinarias COVID-19 y expedientes de gasto tramitados (2020), en web de Ayuntamiento de Madrid. Fecha de consulta: 30-08-20. URL: https://transparencia. madrid.es/portales/transparencia/es/Portada/Informacion-Covid-19/Actuaciones-presupuestarias-COVID-19/?vgne $x t f m t=$ default\&vgnextoid=32b760ff77781710 VgnVCM2000001f4a900aRCRD\&vgnextchannel=e8d97cbbafd71710 VgnVCM2000001f4a900aRCRD.

El interés público de los datos personales en tiempos de COVID-19 (2020), en Hay Derecho. Fecha de consulta: 06-0920. URL: https://hayderecho.expansion.com/2020/04/10/el-interes-publico-de-los-datos-personales-en-tiemposdel-covid-19/.

EI IMV Ilega a menos del $1 \%$ de los hogares que lo piden (2020), en El Confidencial. Fecha de consulta: 22-0820. URL: https://www.elconfidencial.com/espana/2020-08-19/imv-hogares-espana-pobreza-ingreso-minimovital_2718159/.

El Ministerio de Inclusión mejora los procedimientos del Ingreso Mínimo Vital. (2020), en Revista de la Seguridad Social. Fecha de consulta: 23-08-20. URL: https://revista.seg-social.es/2020/09/22/el-ministerio-de-inclusionmejora-los-procedimientos-del-ingreso-minimo-vital/. 
El exceso de muertes en la crisis del coronavirus se eleva hasta los 43.000 muertos (2020), en El País. Fecha de consulta: 02-08-2020. URL: https://elpais.com/sociedad/2020/05/27/actualidad/1590570927 371193.htm/.

Escrivá admite que la Seguridad Social sufra falta de personal y avanza refuerzos (2020), en El Confidencial. Fecha de consulta: 09-08-20. URL: https://www.elconfidencial.com/economia/2020-08-06/escriva-seguridad-social-faltapersonal_2707275/.

Estrategia Española de l+D+l en Inteligencia Artificial (2019). Madrid: Ministerio de Ciencia, Innovación y Universidades. Fecha de consulta: 29-08-2020. URL: https://www.ciencia.gob.es/stfls/MICINN/Ciencia/Ficheros/ Estrategia_Inteligencia_Artificial_IDI.pdf.

GAEBLER, T.; OSBORNE, D. (1992): La reinvención del gobierno: la influencia del espíritu empresarial en el sector público. Barcelona. Ediciones Paidós.

GRASA, R. (2005): "Instituciones para una cooperación al desarrollo de calidad", en Revista CIDOB d'Afers Internacionals, núm. 72, págs. 85-95.

Global Risks Report (2019): Geneva: World Economic Forum. Fecha de consulta: 02-09-20. URL: http://www3. weforum.org/docs/WEF_Global_Risks_Report_2019.pdf.

I Barómetro COVID-19 y Märketing (2020): Madrid: Good Rebels. Fecha de consulta: 16-09-20. URL: https://www. goodrebels.com/wp-content/uploads/2020/09/5\%C2\%AA-oleada-Baro\%CC\%81metro-Covid-y-Marketing-_GoodRebels_VF-1.pdf.

Informe anual de progreso de la Comisión para la Reforma de las Administraciones Públicas (2016). Madrid: Oficina para la Ejecución de la Reforma de la Administración. Fecha de consulta: 28-08-20. URL: https://transparencia.gob. es/transparencia/dam/jcr:7b44c3a3-9e15-4b50-98d0-47b8744c04c7/CORA-informe-anualprogreso-2016-02.pdf.

Informe Estadística de Cuentas Trimestrales no Financieras de los Sectores Institucionales. Cuarto trimestre de 2018 (2019). Madrid: Instituto Nacional de Estadística. Fecha de consulta: 12-08-20. URL: https://www.ine.es/daco/ daco42/ctnfsi/ctnfsi0418.pdf.

KEOHANE, R.; KING, G.; VERBA, S. (2000): El diseño de la investigación social. Madrid: Ciencias Sociales Alianza Editorial.

KUHN, T. (1970): The structure of scientific revolutions. Chicago: University of Chicago Press.

La Administración se reservará gran parte de los fondos de digitalización (2020), en El Economista. Fecha de consulta: 21-09-20. URL: https://www.eleconomista.es/empresas-finanzas/noticias/10767163/09/20/La-Administracion-sereservara-gran-parte-de-los-fondos-de-digitalizacion.html.

La evolución del empleo en las Administraciones públicas durante la última década. Boletín Económico 4/17. (2017). Madrid: Banco de España. Fecha de consulta: 02-09-20. URL: https://www.bde.es/f/webbde/SES/Secciones/ Publicaciones/InformesBoletinesRevistas/NotasEconomicas/T4/fich/bene1704-nec12.pdf.

La Comunidad de Madrid también limita sus servicios de transparencia durante el estado de alarma (2020), en $A B C$. Fecha de consulta: 09-09-20. URL: https://www.abc.es/espana/abci-comunidad-madrid-tambien-limita-serviciostransparencia-durante-estado-alarma-202004280208_noticia.html.

LASSWELL, H.; LERNER, D. (1951): The policy sciences: recent development scope and method. Stanford, CA: Stanford University Press.

LONGO, F. (2019): "La Administración pública en la era del cambio exponencial. Hacia una gobernanza exploratoria", en Revista Vasca de Gestión de Personas y Organizaciones Públicas, núm. 3/2019, págs. 52-73.

LONGO, F. (2008): "Introducción”, en LONGO, F. e YSA, T.: Los escenarios de la gestión pública del siglo XXI, págs. 15-35. Barcelona. Generalitat de Catalunya.

MARGETTS, H.; DUNLEAVY, P. (2013): "The second wave of digital-era governance: a quasi-paradigm for government on the web", en Philosophical Transactions of the Royal Society of London, vol. 371, núm. 1.987, págs. 1-17. DOI: https://doi.org/10.1098/rsta.2012.0382.

MAYNTZ, R. (1980): Sociología de la organización. Madrid: Alianza Universal.

MEIJER, A.; WEBSTER, W. (2020): "The COVID-19 crisis and the information polity: An overview of responses and discussions in twenty-one countries from six continents", en Information Polity, núm. 25, págs. 243-274. DOI: https://doi.org/10.3233/IP-200006.

MENDOZA, X.; VERNIS, A. (1995): “Una aproximación a la conceptualización del nuevo rol del Estado: el Estado relacional”, en Reforma y Democracia, núm. 44, págs. 115-144.

MINTZBERG, H. (1984): La estructuración de las organizaciones. Madrid: Ed. Ariel.

MOLINAS, C. (2013): Qué hacer con España. Barcelona: Destino Editorial.

OCDE (2017): Recommendation of the Council on public integrity. Paris: OECD Publishing. Fecha de Consulta: 24-0820. URL: https://www.oecd.org/gov/ethics/recomendacion-sobre-integridad-es.pdf.

OLMEDA, J. A. (1999): Ciencia de la Administración. Teoría de la Administración y Gestión Pública, vol. I. Madrid: UNED.

O'REILLY, T. (2011): “Government as a platform”, en Innovations, vol. 6, núm. 1, págs. 13-40. DOI: https://doi. org/10.1162/INOV_a_00056.

Plan de Impulso de las Tecnologías del Lenguaje (2015): Madrid: Ministerio de Energía, Turismo y Agenda Digital. Fecha de consulta: 03-09-20. URL: https://www.plantl.gob.es/tecnologias-lenguaje/PTL/ Bibliotecaimpulsotecnologiaslenguaje/Detalle\%20del\%20Plan/Plan-Impulso-Tecnologias-Lenguaje.pdf. 
Plan de Transformación Digital de la Administración General del Estado y sus Organismos Públicos (2015): Madrid: Ministerio de Hacienda y Administraciones públicas. Fecha de consulta: 05-09-2020. URL: https:// administracionelectronica.gob.es/pae_Home/pae_Estrategias/Estrategia-TIC-AGE.html.

Plan España Digital 2025 (2020): Madrid: Gobierno de España. Fecha de consulta: 12-09-20. URL: https://www. lamoncloa.gob.es/presidente/actividades/Documents/2020/230720-Espa\%C3\%B1aDigital_2025.pdf.

Plan para la transición hacia una nueva normalidad (2020). Madrid: Ministerio de Sanidad. Fecha de consulta: $01-$ 09-20. URL: https://www.mscbs.gob.es/profesionales/saludPublica/ccayes/alertasActual/nCov/documentos/ PlanTransicionNuevaNormalidad.pdf.

Por un sector público capaz de liderar la recuperación (2020), en Agenda Pública. Fecha de consulta: 09-09-20. URL: http://agendapublica.elpais.com/por-un-sector-publico-capaz-de-liderar-la-recuperacion/.

RICO, A.; BLACKEY, E. (2012): "Impacto de la crisis en sanidad: recortes estructurales asimétricos con altos costes diferidos", en Informe España 2012. Madrid: Fundación Encuentro.

SABATIER, P.; MAZMANIAN, D. (1981): Effective policy implementation. Washington D. C.: Heat and Company.

SALAZAR, C. (2009): "La evaluación y el análisis de políticas públicas", en Revista Opera, núm. 9, págs. 23-51.

SCHWAB, K. (2016): La cuarta revolución industrial. Barcelona: Debate Editorial.

TALEB, N. (2012): El cisne negro. Madrid. Paidós.

The future of government: lessons learned from around the world (2011). Geneva: World Economic Forum. Fecha de consulta: 27-07-20. URL: https://www. weforum.org/agenda/2011/06/the-future-of-government-lessons-learnedfrom-around-the-world/.

VALENZUELA, R. (2013): "Delimitar Gobierno Abierto para ampliar la colaboración con una sociedad más abierta”, en Estado, Gobierno y Gestión Pública, núm. 21, págs. 157-158. DOI: https://doi.org/10.5354/0717-6759.2013.29403.

VILLORIA, M. (2016): "Transparencia y justicia abierta”, en // Curso de Verano en la Universidad Menéndez Pelayo: Una Justicia para el futuro. Santander: Universidad Menéndez Pelayo.

VILLORIA, M. (2011): “¿Más libertad o más felicidad? El buen gobierno del siglo XXI”, en Revista del CLAD Reforma y Democracia, núm. 51, págs. 5-44.

WHO Coronavirus Disease Dashboard, en web de Organización Mundial de la Salud. Fecha de consulta: 16-09-20. URL: https://covid19.who.int/. 